\title{
DUnisul
}

\section{INFLUÊNCIA DE FATORES ABIÓTICOS NO RENDIMENTO DO ÓLEO ESSENCIAL DA Gallesia integrifolia (SPRENG.) HARMS}

\author{
INFLUENCE OF ABIOTIC FACTORS OVER THE GALLESIA \\ INTEGRIFOLIA ESSENTIAL OIL EXTRACTION YIELD
}

Carlos Henrique Furtado ${ }^{1}$; Andressa Roberta Carneiro²; Zilda Cristiani Gazim ${ }^{3}$; Ary Fernandes Junior $^{4}$; Stella Alonso Rocha ${ }^{5}$; Keila Fernanda Raimundo ${ }^{6}$; Otávio Akira Sakai ${ }^{7}$

1. Instituto Federal do Paraná - IFPR - Campus Avançado Goioerê; E-mail: carlos.furtado@ifpr.edu.br

2. Mestranda do Programa em Sustentabilidade - IFPR - Campus Umuarama; E-mail: andressa_rcarneiro@hotmail.com

3. Universidade Paranaense - UNIPAR; E-mail: cristianigazim@ prof.unipar.br

4. Universidade Estadual Paulista - UNESP - Campus Júlio de Mesquita Filho; E-mail: ary.fernandes@unesp.br

5. Instituto Federal do Paraná - IFPR - Campus Umuarama; E-mail: stella.rocha@ifpr.edu.br

6. Instituto Federal do Paraná - IFPR - Campus Umuarama; E-mail: keila.raimundo@ifpr.edu.br

7. Instituto Federal do Paraná - IFPR - Campus Umuarama; E-mail: otavio.sakai@ifpr.edu.br

\section{RESUMO}

O presente trabalho teve como objetivo avaliar a relação entre o rendimento do óleo essencial obtido das folhas e flores de espécimes da Gallesia integrifolia, localizados no meio ambiente rural na cidade de Icaraíma-PR e distrito de Porto Camargo -PR, com a composição química de seus solos. O processo de extração utilizado foi a hidrodestilação em aparelho Clevenger. Nos locais estudados, quatro amostras de solo foram coletadas e encaminhadas para um laboratório de análise de solos. Os resultados revelaram diferenças significativas entre os micronutrientes - manganês e ferro e entre os macronutrientes as diferenças mais significativas encontradas foram entre os elementos fósforo, ferro e enxofre. Dos 30 parâmetros analisados, $60 \%$ apresentaram menores quantidades no solo de Porto Camargo. Em relação aos rendimentos dos óleos essenciais das flores, a espécie de Porto Camargo apresentou rendimento superior ao de Icaraíma. Fato que pode estar associado a insuficiência de nutrientes presente no solo.

\section{PALAVRAS CHAVES}

Gallesia integrigolia; Óleo Essencial; Hidrodestilação; Solos; Organossulfurados 


\section{ABSTRACT}

This study aimed to evaluate the relationship between the yield of essential oil obtained from the leaves and flowers of specimens of Gallesia integrifolia, located in the rural environment in the city of Icaraima-PR and Porto Camargo-PR district, along with the chemical composition of their soils. The extraction process used was hydrodistillation in a Clevenger apparatus. In the places that were studied, four soil samples were collected and sent to a soil analysis laboratory. The results revealed significant differences between the micronutrients- manganese and iron, and among the macronutrients, the most significant differences were found between the elements phosphorus, iron and sulfur. The soil in Porto Camargo presented lower amounts in $60 \%$ of the 30 parameters that were analyzed. Regarding the yield of the extraction of essential oil from the flowers, the Porto Camargo species showed a higher yield than that of Icaraima. This fact may be associated with insufficient nutrients present in the soil.

\section{KEY WORDS}

Gallesia integrigolia; Essential Oil; Hydrodistillation; Soils; Organosulfur

\section{INTRODUÇÃO}

No Brasil, há uma estimativa de ocorrência de 50.000 espécies de plantas superiores, que fornecem madeira, celulose, alimentos, óleos vegetais e óleos essenciais. O Brasil situa-se em uma posição de destaque sendo considerado o país com a maior riqueza de plantas no mundo (FIORAVANTI, 2016). Entretanto, apenas $8 \%$ das espécies vegetais da flora brasileira foram estudadas em busca de compostos bioativos e apenas 1.100 espécies vegetais desta flora foram avaliadas em suas propriedades medicinais (SIMÕES, et al., 2003).

A espécie vegetal Gallesia integrifolia (Spreng.) Harms, pertencente à família Phytolacaceae e conhecida popularmente como pau d'alho, é encontrada nas regiões brasileiras como região Amazônica, Cerrado, Mata Atlântida e Pantanal (NEGRELLE, 2016). No entanto, Carneiro et al., (2020) reportaram a presença do pau d'alho no Parque Nacional de Ilha Grande, situado no estado do Paraná, sendo uma unidade de conservação brasileira de proteção integral à natureza.

Neste contexto, o pau d'alho está distribuído em diversas regiões do Brasil e as características de cada região, como o clima, solo, pluviometria e incidência solar, podem influenciar nos constituintes orgânicos presentes no óleo essencial (OE). Palazzolo et al., 
(2013) informam que os OEs adquirem variações em relação à quantidade, qualidade e composição química, conforme o tipo de solo, clima, órgão da planta, idade e estágio do ciclo vegetativo. Além disso, os fatores abióticos, como o estresse hídrico, podem afetar muito a concentração de metabólitos secundários, bem como as características morfológicas e fisiológicas (REBEY et al., 2012).

A G. integrifolia é reconhecida como sendo de grande importância para área medicinal como antiinflamatória e capacidade antimicrobiana (ARUNACHALAM et al., 2016; BORTOLUCCI et al., 2021), além disso apresenta atividade contra larvas do Aedes aegypti e carrapato bovino (DIAS et al., 2018; RAIMUNDO et al., 2021). O OE dos frutos de $G$. integrifolia tem alta atividade fungicida conforme os resultados publicados por Raimundo et al. (2018) podendo servir como aplicações futuras na redução do uso de fungicidas sintéticos. Estudos realizados por Bortolucci et al., (2021), utilizando o OE dos frutos, indicaram atividade antiinflamatória e células antitumoral.

Além disso, os estudos conduzidos por Raimundo et al., (2017), mostraram a presença de compostos organossulfurados no $\mathrm{OE}$ dos frutos de G. integrifolia, dentre os quais estão o metanotiol ou metil mercaptano $\left(\mathrm{CH}_{3} \mathrm{SH}\right)$, o 3,6-dithia-1,8-octanodiol $\left(\mathrm{C}_{6} \mathrm{H}_{14} \mathrm{O}_{2} \mathrm{~S}_{2}\right)$ e o 2,3,5-trithiahexano $\left(\mathrm{C}_{3} \mathrm{H}_{8} \mathrm{~S}_{3}\right)$. Sendo assim, o objetivo deste estudo foi verificar a influência do solo no rendimento do OE de folhas e flores da G. integrifolia.

\section{MATERIAIS E MÉTODOS}

\subsection{Material vegetal e local experimental}

As folhas e flores de G. integrifolia foram coletadas a partir de dois espécimes, sendo um localizado na zona rural do município de Icaraíma (IC), Paraná, Brasil (coordenadas 23 - 22'56.3 "S 53 ○ 37'57.2" W) e outro no distrito de Porto Camargo (PC), Paraná, Brasil (coordenadas $23^{\circ} 22^{\prime} 00.4^{\prime S S} 53^{\circ} 45^{\prime} 10.3^{\prime \prime}$ ). Este último está localizado dentro da APA Área de Proteção Ambiental das Ilhas e Várzeas do Rio Paraná, uma unidade de uso sustentável, administrada pelo Instituto Chico Mendes de Conservação da Biodiversidade 


\section{sunisul}

- ICMBio. A autorização concedida pelo Instituto Chico Mendes para a coleta desses materiais encontra-se no registro SISbio n ${ }^{\circ}$ 66717-1, bem como no SISGEN A731238. A coleta das folhas foi realizada em fevereiro de 2019 e das flores em maio de 2019.

\subsection{Composição do solo}

A profundidade de coleta de solo foi de $40 \mathrm{~cm}$ e ocorreu em 04 pontos considerando o raio da distância entre o tronco da árvore e a sua copa projetada no plano do solo. Desta forma, foram coletadas quatro amostras de solo onde está o indivíduo de Porto Camargo e o da zona rural de Icaraíma. As análises de solo foram realizadas no Laboratório Santa Rita para análises de solo, plantas e fertilizantes, na cidade de Mamborê, Paraná, Brasil. A técnica Espectrofotometria de absorção atômica foi utilizada para determinação do Cálcio e Magnésio e a Espectrometria de emissão atômica em plasma (ICP óptico), para quantificação dos elementos Potássio, Cobre, Ferro, Manganês, Zinco e Enxofre. O método colorimétrico foi utilizado para determinação do teor de Carbono orgânico do solo e a Colorimetria da azometina-H para quantificação de Boro nas amostras de solo. Os teores de fósforo foram quantificados por colorimetria do ortofosfórico. Por fim, o $\mathrm{pH}$ foi determinado pelo método eletrométrico e acidez potencial - Método indireto do SMP. Todos as análises citadas seguiram os métodos de Raij, et al., (2001) e EMBRAPA (1997).

\subsection{Rendimento do óleo essencial}

O óleo essencial das folhas e flores da G. Integrifolia foi extraído por hidrodestilação utilizando o aparelho Clevenger, no Laboratório de Química do Instituto Federal do Paraná

- Campus Umuarama. Para cada estrutura vegetal, pesou-se uma amostra de aproximadamente $450 \mathrm{~g}$, que foram trituradas, colocadas em um balão e, então, completando com água até sua capacidade de 3L. O período de extração foi de 3 horas.

O solvente orgânico n-hexano (apolar) foi utilizado para separar, por afinidade química, o hidrolato (polar) e óleo (apolar). Na sequência, essa mistura foi filtrada com papel filtro 
contendo sulfato de sódio anidro (polar), afim de garantir a separação total entre óleo e o hidrolato. As amostras foram armazenadas e refrigeradas para ocorrer a lenta evaporação do n-hexano. Para cada espécie vegetal, a extração foi realizada em triplicata (RAIMUNDO et al. 2018).

\section{RESULTADOS E DISCUSSÃO}

Os resultados das análises de solo retiradas de cada local, estão descritos nas Tabelas 1 e 2, Porto Camargo (PC) e Icaraíma (IC), respectivamente.

Tabela 1: Análise de solo de Porto Camargo - PR.

\begin{tabular}{|c|c|c|}
\hline ELEMENTOS & RESULTADO & UNIDADE \\
\hline 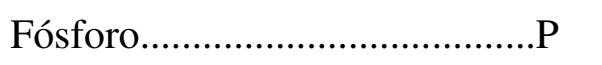 & 8,17 & $\mathrm{mg} / \mathrm{dm}^{3}$ \\
\hline Carbono orgânico Total. .............. & 6,97 & $\mathrm{~g} / \mathrm{dm}^{3}$ \\
\hline $\mathrm{pH}$ em $\mathrm{CaCl}_{2} \ldots \ldots \ldots \ldots \ldots \ldots \ldots$ & 4,82 & \\
\hline Alumínio ……...................... $\left(\mathrm{Al}^{+3}\right)$ & 0,00 & $\mathrm{Cmol}_{\mathrm{c}} / \mathrm{dm}^{3}$ \\
\hline Acidez potencial............ $\left(\mathrm{H}^{+}+\mathrm{Al}^{+3}\right)$ & 3,18 & $\mathrm{Cmol}_{\mathrm{c}} / \mathrm{dm}^{3}$ \\
\hline Cálcio + Magnésio...( $\left(\mathrm{Ca}^{+2}+\mathrm{Mg}^{+2}\right)$ & 1,96 & Cmold $/ \mathrm{dm}^{3}$ \\
\hline Cálcio................................. & 1,35 & $\mathrm{Cmol}_{c} / \mathrm{dm}^{3}$ \\
\hline Magnésio.............................. $\left(\mathrm{Mg}^{+2}\right)$ & 0,61 & $\mathrm{Cmol}_{\mathrm{c}} / \mathrm{dm}^{3}$ \\
\hline Potássio & 0,11 & $\mathrm{Cmol}_{\mathrm{c}} / \mathrm{dm}^{3}$ \\
\hline Soma de bases.......................... & 2,07 & $\mathrm{Cmol}_{\mathrm{c}} / \mathrm{dm}^{3}$ \\
\hline C.T.C ${ }^{1}$ & 5,25 & $\mathrm{Cmol}_{\mathrm{c}} / \mathrm{dm}^{3}$ \\
\hline Saturação por bases................V\% & 39,43 & $\%$ \\
\hline Saturação por alumínio...........m\% & 0,00 & $\%$ \\
\hline Saturação por cálcio..........\% $\mathrm{Ca}^{+2}$ & 25,71 & ${ }^{2}$ Saturação complexo de troca \\
\hline Saturação por magnésio. $\% \mathrm{Mg}^{+2}$ & 11,62 & Saturação complexo de troca \\
\hline Saturação por potássio.........\% $\mathrm{K}^{+}$ & 2,1 & Saturação complexo de troca \\
\hline Cálcio / Magnésio.............. a / Mg & 2,21 & ${ }^{3}$ Relações \\
\hline Cálcio / Potássio.................... / K & 12,27 & Relações \\
\hline Magnésio / Potássio...........Mg / K & 5,55 & Relações \\
\hline 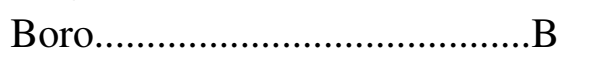 & 0,19 & $\mathrm{mg} / \mathrm{dm}^{3}$ \\
\hline Cobre........................................... & 2,41 & $\mathrm{mg} / \mathrm{dm}^{3}$ \\
\hline 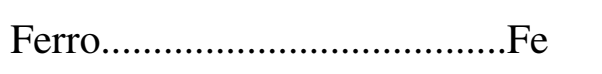 & 15,3 & $\mathrm{mg} / \mathrm{dm}^{3}$ \\
\hline Manganês....................................... & 157,5 & $\mathrm{mg} / \mathrm{dm}^{3}$ \\
\hline \multicolumn{3}{|c|}{ 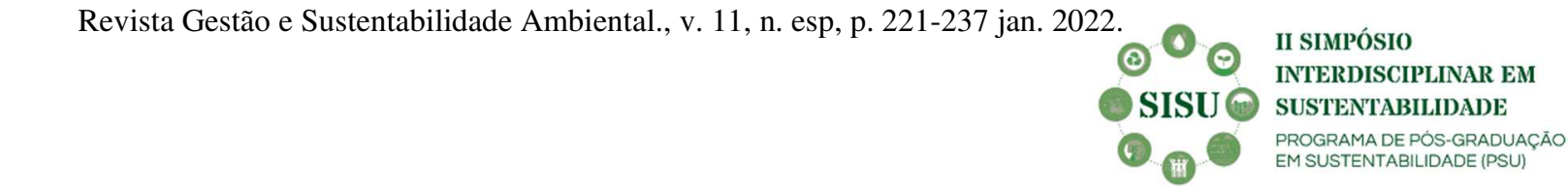 } \\
\hline
\end{tabular}


Zinco .Zn

Enxofre $\left(\mathrm{S}-\mathrm{SO}_{4}{ }^{-2}\right)$

Fósforo Remanescente...P-Rem

$\mathrm{pH}$ em $\mathrm{H} 2 \mathrm{O}$.

Argila

Silte

Areia.
4,76

5,93

$\mathrm{Nc}$

$\mathrm{Nc}$

101

25

874 $\mathrm{mg} / \mathrm{dm}^{3}$

$\mathrm{mg} / \mathrm{dm}^{3}$

$\mathrm{mg} / \mathrm{L}$

$\mathrm{g} / \mathrm{kg}$

$\mathrm{g} / \mathrm{kg}$

$\mathrm{g} / \mathrm{kg}$

Tabela 2: Análise de solo de Icaraíma - PR.

\begin{tabular}{|c|c|c|}
\hline ELEMENTOS & RESULTADO & UNIDADE \\
\hline 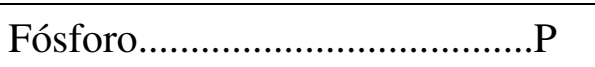 & 45,98 & $\mathrm{mg} / \mathrm{dm}^{3}$ \\
\hline Carbono orgânico Total. ...........C & 7,07 & $\mathrm{~g} / \mathrm{dm}^{3}$ \\
\hline $\mathrm{pH}$ em $\mathrm{CaCl}_{2} \ldots \ldots \ldots \ldots \ldots \ldots \ldots \ldots$ & 5,31 & \\
\hline Alumínio ................................ $\left(\mathrm{Al}^{+3}\right)$ & 0 & $\mathrm{Cmol}_{\mathrm{c}} / \mathrm{dm}^{3}$ \\
\hline Acidez potencial.......... $\left(\mathrm{H}^{+}+\mathrm{Al}^{+3}\right)$ & 2,54 & $\mathrm{Cmol}_{\mathrm{c}} / \mathrm{dm}^{3}$ \\
\hline Cálcio + Magnésio. $\left(\mathrm{Ca}^{+2}+\mathrm{Mg}^{+2}\right)$ & 2,28 & $\mathrm{Cmol}_{\mathrm{c}} / \mathrm{dm}^{3}$ \\
\hline Cálcio................................. & 1,61 & $\mathrm{Cmol}_{\mathrm{c}} / \mathrm{dm}^{3}$ \\
\hline Magnésio............................ $\left(\mathrm{Mg}^{+2}\right)$ & 0,65 & $\mathrm{Cmol}_{\mathrm{c}} / \mathrm{dm}^{3}$ \\
\hline Potássio ................................... $\left(\mathrm{K}^{+}\right)$ & 0,32 & $\mathrm{Cmol}_{\mathrm{c}} / \mathrm{dm}^{3}$ \\
\hline Soma de bases.......................SB & 2,6 & $\mathrm{Cmol}_{\mathrm{c}} / \mathrm{dm}^{3}$ \\
\hline (n)......................... & 5,14 & $\mathrm{Cmol}_{\mathrm{c}} / \mathrm{dm}^{3}$ \\
\hline Saturação por bases...............V\% & 50,58 & $\%$ \\
\hline Saturação por alumínio............m\% & 0 & $\%$ \\
\hline Saturação por cálcio...........\% $\% \mathrm{Ca}^{+2}$ & 31,71 & ${ }^{2}$ Saturação complexo de troca \\
\hline Saturação por magnésio... $\% \mathrm{Mg}^{+2}$ & 12,65 & Saturação complexo de troca \\
\hline Saturação por potássio........... $\% \mathrm{~K}^{+}$ & 6,23 & Saturação complexo de troca \\
\hline Cálcio / Magnésio.............Ca / Mg & 2,51 & ${ }^{3}$ Relações \\
\hline Cálcio / Potássio..................Ca / K & 5,09 & Relações \\
\hline Magnésio / Potássio.............Mg / K & 2,03 & Relações \\
\hline 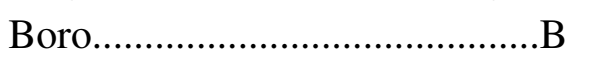 & 0,26 & $\mathrm{mg} / \mathrm{dm}^{3}$ \\
\hline 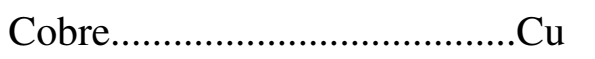 & 2,78 & $\mathrm{mg} / \mathrm{dm}^{3}$ \\
\hline 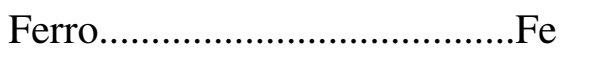 & 80,2 & $\mathrm{mg} / \mathrm{dm}^{3}$ \\
\hline 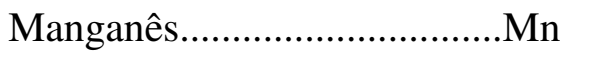 & 73,3 & $\mathrm{mg} / \mathrm{dm}^{3}$ \\
\hline 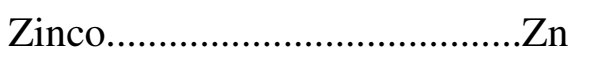 & 5,07 & $\mathrm{mg} / \mathrm{dm}^{3}$ \\
\hline Enxofre ......................... (S-SO $\left.{ }_{4}^{-2}\right)$ & 3,17 & $\mathrm{mg} / \mathrm{dm}^{3}$ \\
\hline Fósforo Remanescente...P-Rem & $\mathrm{Nc}$ & $\mathrm{mg} / \mathrm{L}$ \\
\hline 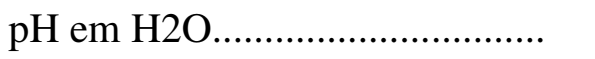 & $\mathrm{Nc}$ & \\
\hline
\end{tabular}

Revista Gestão e Sustentabilidade Ambiental., v. 11, n. esp, p. 221-237 jan. 2022.

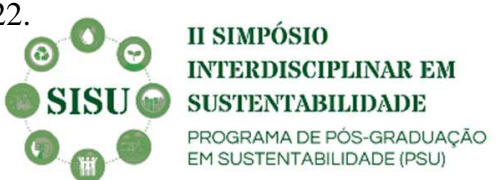


Argila

Silte.

Areia.

.
86

30

884 $\mathrm{g} / \mathrm{kg}$

$\mathrm{g} / \mathrm{kg}$

$\mathrm{g} / \mathrm{kg}$

IIndica a capacidade do solo em reter nutrientes (CTC - Capacidade de Troca Catiônica). ${ }^{2}$ Indica a quantidade do nutriente em relação a CTC do solo. ${ }^{3}$ Indica a proporção entre si, da presença dos elementos no solo.

Na figura 01, a partir das tabelas 1 e 2 apresentamos alguns parâmetros analisados nos solos PC e IC. Destacamos nesta figura, aqueles que apresentaram diferenças acima dos 20 por cento entre os solos, sendo: enxofre, manganês, ferro, cálcio, saturação por bases e fósforo.

\begin{tabular}{|c|c|}
\hline \multicolumn{2}{|c|}{$\begin{array}{l}\text { Diferenças significativas dos solos de Icaraíma (IC) - vermelho e Porto Camargo } \\
\text { (PC) - Preto }\end{array}$} \\
\hline $\begin{array}{r}\text { Enxofre / IC / mg/dm3 } \\
\text { Enxofre / PC / mg/dm3 } \\
\text { Manganês / IC / mg/dm3 } \\
\text { Manganês / PC / mg/dm3 } \\
\text { Ferro / IC / mg/dm3 } \\
\text { Ferro / PC / mg/dm3 } \\
\text { Cálcio /Potássio / IC } \\
\text { Cálcio /Potássio / PC } \\
\text { Saturação por bases V\%/IC } \\
\text { Saturação por bases V\%/PC } \\
\text { Fósforo / IC / mg/dm3 } \\
\text { Fósforo / PC / mg/dm3 }\end{array}$ & 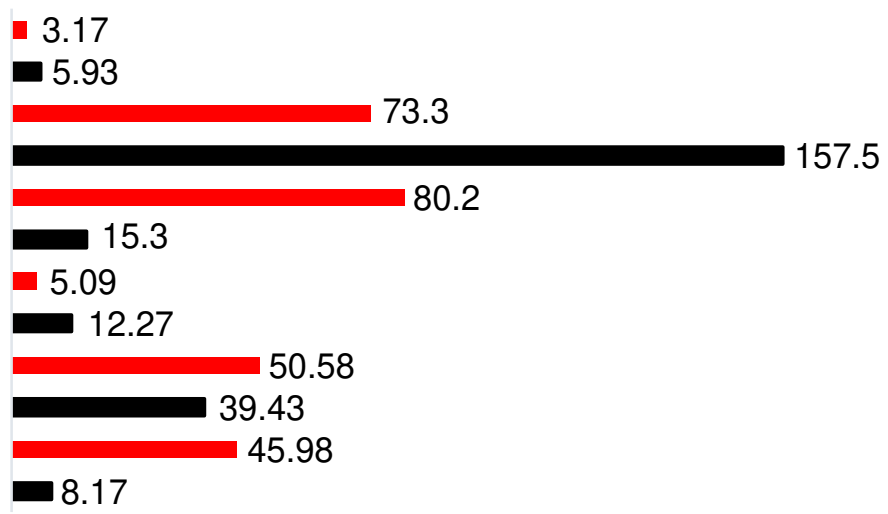 \\
\hline
\end{tabular}

FIGURA 01: Diferenças entre os parâmetros enxofre, manganês, ferro, cálcio, saturação por base e fósforo dos solos dos espécimes de G. integrifolia. Fonte: Autores

No solo onde se localiza os espécimes de PC e IC o teor de Fósforo encontrado foi $8,17 \mathrm{mg} / \mathrm{dm}^{3}$ e $45,98 \mathrm{mg} / \mathrm{dm}^{3}$, respectivamente. Na literatura não se encontra disponibilizado a quantidade adequada padrão de Fósforo no solo, pois isso depende de fatores como a textura do solo, o teor de argila, além do tipo de cultura para a qual o solo será utilizado. Percebe-se que no solo da zona rural de Icaraíma, o teor de Fósforo é aproximadamente 5,6 vezes maior. Para Fink et al., (2016), a principal causa da baixa disponibilidade desse nutriente para as plantas é a grande estabilidade dos fosfatos na fase sólido do solo, decorrente da formação de compostos que se ligam aos colóides, 
especialmente aos oxi-hidróxidos de Fe e Al, constituintes da fração de argila que mais adsorvem o elemento $\mathrm{P}$.

Esse elemento é absorvido pela planta na forma de $\mathrm{H}_{2} \mathrm{PO}_{4}{ }^{-1}$ e sua acumulação ocorre nas raízes, pela qual-é transportado até o xilema através do simplasto e chega às folhas ou às regiões de crescimento, sendo juntamente com o nitrogênio o elemento mais prontamente redistribuído (JÚNIOR et al., 2009).

Conforme Zambolim e Ventura (1993), o Fósforo é um dos treze elementos minerais essenciais para o crescimento das plantas. Os demais elementos são Nitrogênio, Potássio, Cálcio, Magnésio, Enxofre, Manganês, Zinco, Cobre, Boro, Ferro, Molibdênio e Cloro. Relataram ainda que a deficiência ou excesso de um elemento influencia grandemente na atividade dos outros com efeito direto no metabolismo da planta. Os autores destacaram que a simples presença de um elemento químico no solo não implica necessariamente que ele está suficientemente disponível para o crescimento da planta, e que a sua disponibilidade é função da sua quantidade no solo, sua forma e sua solubilidade, a capacidade assimilativa da planta e o meio ambiente do solo ( $\mathrm{pH}$, umidade e temperatura) (ZAMBOLIM e VENTURA, 1993).

Para Batista et al., (2003), a deficiência no quantitativo de Fósforo poderá ter relação com o estreitamento das folhas, formato reduzido, bordos curvados e ápice voltado para baixo e alteração na cor natural. Os autores relataram ainda que as plantas com deficiência em Fósforo têm o seu crescimento retardado, devido afetar vários processos, como a síntese proteica e de ácidos nucléicos.

Especificamente em relação ao Fósforo, Zambolim e Ventura (1993), informaram que esse elemento aumenta a resistência da planta pelo aumento do teor ou por acelerar a maturação dos tecidos, auxiliando a planta a escapar dos patógenos. De um modo geral, o Fósforo tem sido importante no decréscimo do ataque de fungos. Mas destacaram que em certos casos, o aumento do nível do fósforo pode ter um efeito contrário, ou seja, favorecer o ataque de doenças como a ferrugem.

De fato, a G. integrifolia localizada em PC apresentou folhas menores e coloração menos intensa quando comparadas às folhas do espécime de IC. Além disso, seu tronco 
apresentava uma cavidade oca tomada por fungos, o que pode estar relacionado com alguma deficiência de compostos com ação antifúngica.

Outro elemento químico, o Ferro, obteve-se resultados com diferenças relevantes entre as duas amostras de solo. No solo de IC o valor encontrado foi de 5,2 vezes maior, ou seja, $80,20 \mathrm{mg} / \mathrm{dm}^{3}$ e $15,30 \mathrm{mg} / \mathrm{dm}^{3}$ para PC.

Nas plantas, o elemento Ferro está relacionado a diversas atividades metabólicas, participando da formação de algumas enzimas (catalase, peroxidase, citocromo oxidase e xantina oxidase), além de ser indispensável nos processos de respiração, fotossíntese, fixação de $\mathrm{N}_{2}$ e transferência de elétrons através do ciclismo entre o $\mathrm{Fe}^{2+}$ e $\mathrm{Fe}^{3+}$ (ALEXANDRE et al., 2012).

$\mathrm{Na}$ deficiência de $\mathrm{Fe}$, há menos RNA e menor taxa de síntese de proteínas. 75 por cento do Fe encontra-se nos cloroplastos e, consequentemente, na sua deficiência, ocasiona clorose das folhas jovens, pela diminuição da quantidade de cloroplasto e do teor de clorofila (SFREDO; BORKERT, 2004).

O Manganês foi outro elemento químico que apresentou diferença de teor no solo entre os dois locais. No solo de PC foi encontrado o valor de $157,50 \mathrm{mg} / \mathrm{dm}^{3}$, isso significa uma quantidade aproximadamente 2,1 vezes maior em comparação ao teor do solo em IC, sendo $73,30 \mathrm{mg} / \mathrm{dm}^{3}$.

Para Kirkby e Römheld (2007), o papel mais bem documentado e exclusivo do Mn em plantas verdes é aquele da reação de quebra da molécula da água e do sistema de evolução de $\mathrm{O}_{2}$ na fotossíntese que ocorre nos cloroplastos, denominado reação de Hill. Em decorrência desta função-chave na reação de quebra da água, a deficiência de Mn afeta principalmente a fotossíntese e a evolução de Oxigênio. Até mesmo deficiências leves de Mn afetam a fotossíntese e diminuem o nível de carboidratos solúveis na planta, porém, o re-suprimento deste micronutriente reativa a evolução fotossintética de oxigênio. Quando ocorre deficiência deste micronutriente a reação à luz durante a fotossíntese é seriamente prejudicada e todas as outras reações associadas com o transporte de elétrons também o são. Com deficiência mais severa de Mn, entretanto, ocorre uma quebra na estrutura do cloroplasto que não pode ser revertida. 
O Mn também atua como um importante co-fator para várias enzimas-chave na biossíntese dos metabólitos secundários da planta associados com a via do ácido chiquímico, incluindo aminoácidos aromáticos fenólicos, cumarinas, ligninas e flavonóides (BURNELL, 1988 apud KIRKBY e RÖMHELD, 2007). No mesmo sentido, os autores informaram que concentrações mais baixas de compostos fenólicos, lignina e flavonóides foram detectadas em tecidos deficientes em Mn, o que pode, em parte, ser a causa da maior suscetibilidade a doenças das plantas deficientes neste micronutriente (GRAHAM, 1983 apud KIRKBY e RÖMHELD, 2007).

Teixeira et al., (2005), informaram que em várias funções nas plantas, o Manganês e o Zinco são determinantes ou integrantes de diversos processos, tais como síntese de proteínas, permeabilidade de membranas, absorção iônica, respiração, síntese de amido e controle hormonal. Relataram ainda que, o Manganês está relacionado à formação da lignina, que, por sua vez, é uma das substâncias presentes na parede celular, conferindolhe impermeabilidade (Teixeira et al., 2005).

Com relação ao teor de Enxofre no solo, as análises apontaram $5,93 \mathrm{mg} / \mathrm{dm}^{3}$ no solo onde se localiza o espécime de PC e $3,17 \mathrm{mg} / \mathrm{dm}^{3}$ para o solo onde está localizado o espécime da zona rural de IC. Quanto a presença deste elemento nos vegetais, Viégas et al., (2004), informaram que a sua deficiência pode ocasionar principalmente a clorose nas folhas novas. Os autores informaram ainda que nas folhas verdes a maioria das proteínas estão localizadas nos cloroplastos e nas moléculas da clorofila, e quando as plantas apresentam deficiência em Enxofre, consequentemente, terão menor teor de clorofila apresentando uma coloração verde-pálida. Outros autores como Batista et al., (2003), relataram que a deficiência de Enxofre em plantas de gravioleiras causaram a presença de folhas superiores com deformação e com coloração verde-pálida, tornando-se posteriormente, cloróticas.

Em relação à acidez trocável, representada pelo alumínio $\left(\mathrm{Al}^{3+}\right)$, de acordo com Sobral et al., (2015), a presença deste cátion no solo pode inibir o crescimento radicular e influenciar na disponibilidade de outros nutrientes. Nesta análise de solo, não foi verificado a presença deste elemento na sua forma catiônica; no entanto, para a acidez total ou 
potencial $\left(\mathrm{H}^{+}+\mathrm{Al}^{3+}\right)$, foi encontrado o índice 3,18 e 2,54 $\mathrm{Cmol}_{\mathrm{d}} / \mathrm{dm}^{3}$ para os solos de PC e IC, respectivamente. Para a análise capacidade de troca catiônica (CTC), foi verificado os valores 5,25 e 5,14 $\mathrm{Cmol}_{\mathrm{c}} / \mathrm{dm}^{3}$ para os solos de PC e IC, respectivamente. Esses valores para efeito de análises do solo são considerados médios ou bons; porém, valores abaixo de $5 \mathrm{Cmol}_{\mathrm{c}} / \mathrm{dm}^{3}$ podem indicar baixo teor de argila.

Referente a saturação por bases (V\%), foi verificado 39,43\% para o solo de PC e $50,58 \%$ para o solo do espécime IC. Quando esse valor fica abaixo de 50\%, pode indicar a necessidade de correção; no entanto, conforme a região do país, esses valores podem ser considerados adequados. $\mathrm{O}$ teor de potássio $\left(\mathrm{K}^{+}\right)$fornecidos pela análise foi de 0,11 $\mathrm{Cmol}_{\mathcal{C}} / \mathrm{dm}^{3}$ e 0,32 $\mathrm{Cmol}_{\mathrm{c}} / \mathrm{dm}^{3}$ para os solos de PC e Icaraíma, respectivamente. Para o solo de PC esse valor é considerado médio ou bom, mas para o solo do espécime IC, o valor de $0,32 \mathrm{Cmol}_{\mathrm{c}} / \mathrm{dm}^{3}$ é considerado muito alto.

O teor de argila constatado foi de $101 \mathrm{~g} / \mathrm{kg}$ de solo de PC e $86 \mathrm{~g} / \mathrm{kg}$ de solo de IC. Esses valores indicam que esses solos analisados são considerados arenosos. $\mathrm{O}$ teor de fósforo (P) em solos arenosos é considerado baixo quando fica entre $0-10 \mathrm{mg} / \mathrm{dm}^{3}$. O solo de PC apresentou um teor de $8,17 \mathrm{mg} / \mathrm{dm}^{3}$ e o solo de IC apresentou um teor de 45,98 $\mathrm{mg} / \mathrm{dm}^{3}$, sendo considerado baixo para o solo de PC e muito alto para o solo de IC. Segundo o proprietário, em outras épocas, o local foi utilizado para descartar os resíduos gerados pela criação do bicho da seda, o que pode explicar o alto teor do elemento fósforo no local.

Quanto ao teor de matéria orgânica total ou carbono orgânico total, é considerado baixo quando esse índice fica entre $7-20 \mathrm{~g} / \mathrm{dm}^{3}$. No solo de PC foi verificado o valor de $6,97 \mathrm{~g} / \mathrm{dm}^{3}$ e 7,07 $\mathrm{g} / \mathrm{dm}^{3}$ para o solo de IC. Esses valores podem indicar que os solos analisados contem baixa matéria orgânica. Quanto ao índice de saturação por $\% \mathrm{Ca}^{2+}$, no solo de PC foi constatado o valor de $25,71 \%$, sendo, portanto, um valor considerado baixo. O solo analisado de IC apresentou teor de $31,71 \%$, sendo considerado um índice baixo para médio.

Quanto ao índice de saturação por $\% \mathrm{Mg}^{2+}$ é considerado médio quando fica entre 10 a 15\%. A análise do solo de PC apresentou um índice de 11,62\% e 12,65\% para o solo de IC. Esses valores são considerados próximos do adequado. Para o índice de saturação 
por $\% \mathrm{~K}^{+}$, a análise do solo de PC foi de 2,10\% sendo considerado bom e 6,23\% para o solo de IC, sendo considerado alto. Para a análise da relação $\mathrm{Ca} / \mathrm{Mg}$ é considerado médio ou bom, quando está entre 2 a 5 . Ambos os solos analisados podem ser considerados bom para esta relação, pois os valores verificados foram 2,21 e 2,51 para PC e IC, respectivamente.

Quando a análise considera a relação $\mathrm{Ca} / \mathrm{K}$, o solo de $\mathrm{PC}$ foi considerado estar no padrão médio para alto, pois seu índice foi de 12,27. Para o solo de IC o valor encontrado foi de 5,09 e sendo, portanto, considerado baixo. Para a relação $\mathrm{Mg} / \mathrm{K}$ foi verificado o valor de 5,55 e 2,03 para os solos de Porto Camargo e Icaraíma, respectivamente. Para o primeiro solo o valor é considerado adequado, enquanto que para o segundo solo o valor 2,03 é considerado baixo.

A análise dos micronutrientes ou microelementos, apresentaram os seguintes valores: no solo de PC o teor de Boro (B) foi de $0,19 \mathrm{mg} / \mathrm{dm}^{3}$ e $0,26 \mathrm{mg} / \mathrm{dm}^{3}$ para o solo de IC. Essa quantidade de Boro apresentada é considerada insuficiente para o desenvolvimento das plantas. O solo de Porto Camargo apresentou o teor de Cobre $(\mathrm{Cu})$ $2,41 \mathrm{mg} / \mathrm{dm}^{3}$ e o solo da zona rural de Icaraíma $2,78 \mathrm{mg} / \mathrm{dm}^{3}$. Portanto, esses valores são considerados altos e podem causar fitotoxicidade para a planta, pois o teor considerado próximo do adequado, fica entre 0,5 e $0,8 \mathrm{mg} / \mathrm{dm}^{3}$. Para o elemento Ferro $(\mathrm{Fe})$, no solo de Porto Camargo o teor encontrado foi de $15,30 \mathrm{mg} / \mathrm{dm}^{3}$, sendo considerado baixo podendo induzir uma menor absorção de outros nutrientes. E no solo da zona rural de Icaraíma, o teor deste elemento apresentou um quantitativo de $80,20 \mathrm{mg} / \mathrm{dm}^{3}$. Para o elemento Manganês (Mn), a quantidade considerada próximo do adequado fica na faixa de 15 a 30 $\mathrm{mg} / \mathrm{dm}^{3}$. No solo de Porto Camargo foi encontrado a quantidade de $157,50 \mathrm{mg} / \mathrm{dm}^{3}$, e no solo da zona rural de Icaraíma a quantidade verificada foi de 73,30 mg/dm 3 . Em ambos os solos esse elemento está em excesso e, uma das consequências é interferir no transporte do elemento Ferro no interior do vegetal. Quanto ao elemento Zinco ( $\mathrm{Zn}$ ), a quantidade encontrada no solo de Porto Camargo foi de $4,76 \mathrm{mg} / \mathrm{dm}^{3}$ e no solo da zona rural de Icaraíma foi de $5,07 \mathrm{mg} / \mathrm{dm}^{3}$. Em ambos é considerado um alto índice. 
Para o elemento Cálcio, no solo de Porto Camargo o valor encontrado é de 1,35 $\mathrm{Cmol}_{\mathrm{c}} / \mathrm{dm}^{3}$ sendo considerado baixo. E no solo da zona rural de Icaraíma foi de 1,63 $\mathrm{Cmol}_{\mathrm{c}} / \mathrm{dm}^{3}$, sendo este valor considerado alto.

Quanto ao elemento enxofre $\left(\mathrm{S}_{-} \mathrm{SO}_{4}{ }^{-2}\right)$, no solo de Porto Camargo apresentou o quantitativo de $5,93 \mathrm{mg} / \mathrm{dm}^{3}$, sendo considerado uma quantidade média. No solo da zona rural de Icaraíma, a quantidade foi de $3,17 \mathrm{mg} / \mathrm{dm}^{3}$, sendo considerado uma quantidade muito baixa (VILLAR, 2007; SOBRAL et al., 2015).

Os resultados das análises de elementos químicos presentes nos solos podem contribuir para entender a diferença de rendimento encontrado para as flores dos dois espécimes estudados. Na Figura 2 é demonstrado o rendimento médio do óleo essencial extraído das folhas e flores dos espécimes de Icaraíma e Porto Camargo.

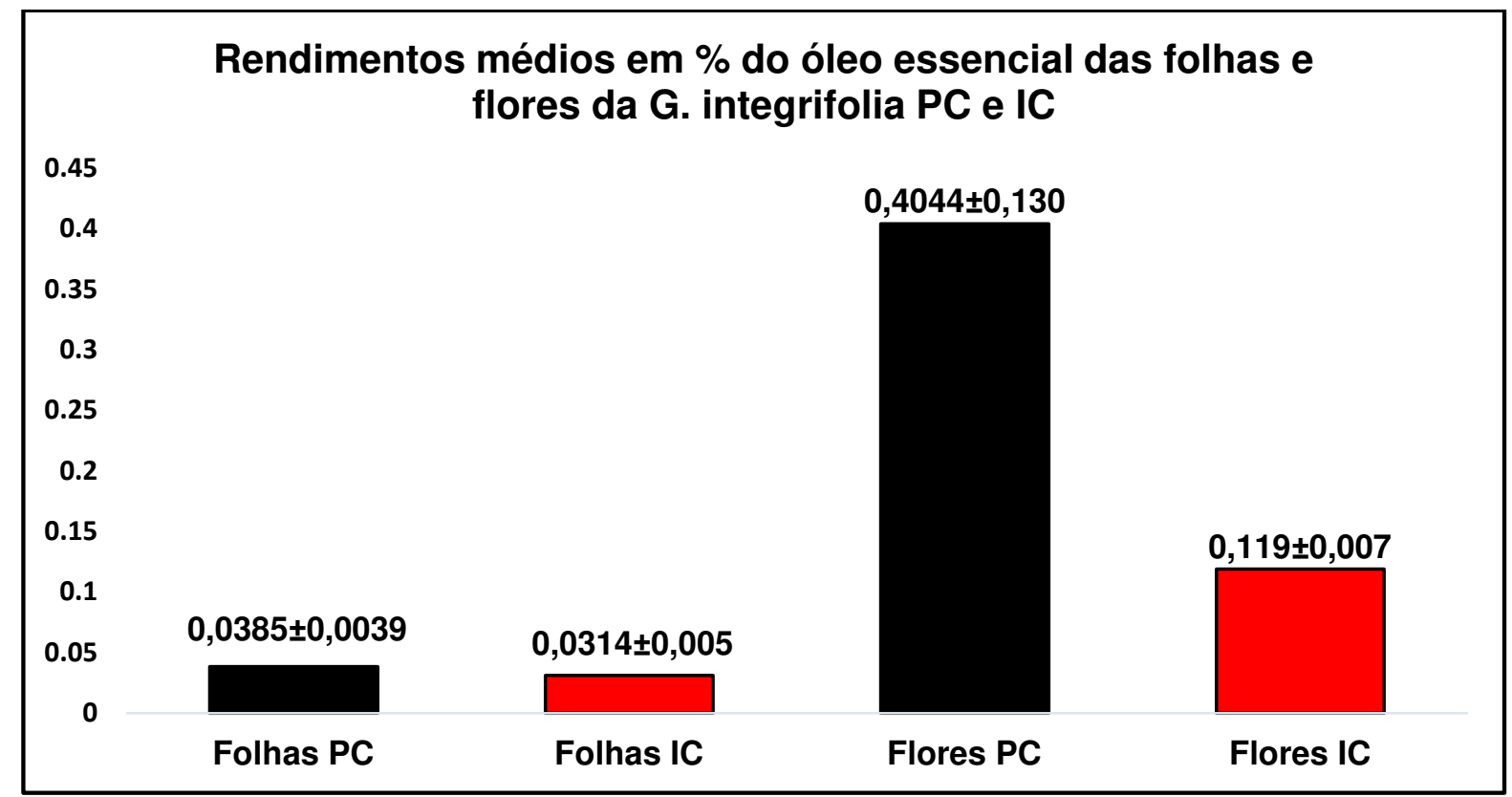

FIGURA 02: Porcentagem do rendimento médio do óleo essencial obtido a partir das folhas e flores de Gallesia integrifolia dos espécimes de Porto Camargo e Icaraíma. Fonte: Autores

O rendimento do OE para as folhas foi de $0,0385 \pm 0,0039 \%$ e $0,0314 \pm 0,005 \%$ para PC e IC, respectivamente. Para as flores podemos verificar que PC produziu cerca de 10 vezes mais $\mathrm{OE}$ em comparação com as folhas e aproximadamente 3,4 vezes mais que o obtido das flores do indivíduo IC. Considerando que as flores são estruturas que darão 


\section{DUnisul}

origem a novos espécimes da espécie, é razoável compreender que nelas, as plantas concentrem substâncias que viabilizem a continuação da espécie através da reprodução.

Os óleos essenciais são metabólitos secundários extraídos de diversas partes de plantas, possuem composição química complexa e garantem aos vegetais, vantagens adaptativas no meio em que estão inseridos. A composição química dos óleos voláteis varia entre as espécies e partes de um mesmo vegetal. Uma mesma espécie botânica pode ser afetada pelo local de cultivo, condições de coleta, estabilização e estocagem, além dos fatores edafoclimáticos (MIRANDA et al., 2016).

Além disso, Palazzolo et al., (2013), ao mencionarem que as plantas são fontes de compostos conhecidos por "metabólitos secundários", destacaram que esses compostos estão diretamente relacionados com o papel defensivo e atrativo nas interações da planta com o meio ambiente, incluindo a relação com outras plantas, com herbívoros, patógenos e polinizadores. Sendo as flores a estrutura que atrai vertebrados e insetos polinizadores, o aroma exalado por essa estrutura, estaria diretamente relacionado com a atração. Nestas partes da planta, pode haver uma maior quantidade de glândulas secretoras de metabólitos secundários.

$\mathrm{Na}$ análise do solo de IC foi encontrado uma menor quantidade do teor de Manganês comparado com PC. Segundo Miranda et al., (2016), esse elemento está relacionado com a eficiência na fotossíntese e tem relação com a biossíntese dos metabólitos secundários. Aliado a isso, os teores de Ca e $\mathrm{S}$ foram também menores no solo de IC em relação a PC. De fato, isso pode ter contribuído para um menor rendimento do óleo essencial das flores de IC. Outra hipótese, provavelmente, é a que as maiores diferenças foram para os elementos $\mathrm{P}$ e Fe. O solo de PC em comparação com o de IC, as análises indicam que para esses elementos, houve a mais significativa diferença, sendo o teor no solo PC cerca de 5 vezes menor. Rodrigues e Carvalho (2001) corroboram e afirmam que estresses nutricionais podem induzir as plantas à maior produção de óleo essencial. 


\section{CONCLUSÃO}

O OE extraído das flores de G. integrifolia apresentou um rendimento cerca de 10 vezes maior quando comparado ao OE extraído das folhas do espécime de Porto Camargo e aproximadamente 3 vezes maior para o espécime na zona rural de Icaraíma.

Os resultados obtidos na análise dos solos demonstraram que a falta e/ou excesso de nutrientes ou ainda, a variação no teor dos principais nutrientes nos solos onde estão os espécimes estudados, pode acarretar diferenças importantes no rendimento do óleo essencial extraído, principalmente das flores da G. integrifolia.

Esse é o primeiro estudo, até o presente momento, sobre a influência da composição química do solo na produção e extração do OE da G. integrifolia.

\section{REFERÊNCIAS}

Alexandre, J. R. et al. Zinco e ferro: de micronutrientes a contaminantes do solo. Natureza on line 10 (1): 23-28, 2012. Disponível em: < https://www.bibliotecaagptea.org.br/agricultura/defesa/artigos/ZINCO $\% 20 \mathrm{E} \% 20 \mathrm{FERRO}$ $\% 20 \% 20$ DE $\% 20$ MICRONUTRIENTES\%20A\%20CONTAMINANTES\%20DO\%20SO LO.pdf >. Acesso em Ago. 2021.

Arunachalam, K. et al. Gallesia integrifolia (Spreng.) Harms: In vitro and in vivo antibacterial actiities and mode of action. Journal of Ethnopharmacology 184:128 - 137, 2016.

Batista, M. M. F. et al. Efeito da omissão de macronutrientes no crescimento, nos sintomas de deficiências nutricionais e na composição mineral em gravioleiras (Annona muricata). Jaboticabal - SP, Rev. Bras. Frutic., v. 25, n. 2, p. 315-318, Ago. 2003. Disponível em: < http://www.scielo.br/pdf/\%0D/rbf/v25n2/a33v25n2.pdf >. Acesso em: Nov. 2019.

Bortolucci et al, Cytotoxicity and anti-inflammatory activities of Gallesia integrifolia (Phytolaccaceae) fruit essential oil, Nat Prod Res. 2021 May 18;1-6. https://doi.org/10.1080/14786419.2021.1925270

Carneiro, A. R.; Furtado, C. H.; Martins, A. P. C.; Zucareli, V.; Martins, D. A.; Sakai, O. A. Application of Gallesia integrifolia (spreng.) harms hydrolate in germination of millet seeds. Revista mundi engenharia, tecnologia e gestão, v. 5, p. 1-11, 2020.

Dias, A. S. et al. In vitro effect of extract of garlic's wood (Gallesia integrifolia Sprengel) Harms on engorged ticks and larvae of Ripicephalus Boophilus microplus. Scientific Electronic Archives Issue, Sci. Elec. Arch. Vol. 11 (5) October 2018. Disponível em: 
$<$ https://www.seasinop.com.br/revista/index.php?journal=SEA\&page=article \&op=view \& path\%5B\%5D=679 >. Acesso em: Ago. 2021.

Embrapa. Centro Nacional de Pesquisa de Solos (Rio de Janeiro, RJ) Manual de métodos de análise de solo. 2.ed.rev.atual. Rio de Janeiro, 1997. 212p. (EMBRAPA - CNPS. Documentos, 1).

Fink, J. R.; Inda, A. V.; Tiecher, T.; Barrón, V. 2016. Iron oxides and organic matter on soil phosphorus availability. Ciência e Agrotecnologia, 40, 369-379. http://dx.doi. org/10.1590/1413- 70542016404023016.

Fioravanti, C. A maior diversidade de plantas do mundo. Revista Pesquisa Fapesp, Ed. 241, mar. 2016.

Júnior, A. B. D. A. et al. Efeito de doses de fósforo no desenvolvimento inicial da mamoneira. Mossoró - RN, Caatinga, v.22, n.1, p.217-221, Jan. / Mar. 2009

Kirkby, E. A.; Römheld. V. Micronutrientes na fisiologia de plantas: micronutrientes na fisiologia de plantas: micronutrientes na fisiologia de plantas: funções, absorção e mobilidade. Tradução: Suzana Oellers Ferreira, Encarte Técnico, Informações Agronômicas, $\mathrm{n}$ 118, Jun. 2007. Disponível em: $<$ http://www.ipni.net/publication/iabrasil.nsf/0/8A79657EA91F52F483257AA10060FAC B/\$FILE/Encarte-118.pdf >. Acesso em: Ago. 2021

Miranda, C. A. S. F. et al. Óleos essenciais de folhas de diversas espécies: propriedades antioxidantes e antibacterianas no crescimento espécies patogênicas. Fortaleza - CE, Revista Ciência Agronômica, v. 47, n. 1, p. 213-220, jan-mar, 2016. Disponível em: < http://www.scielo.br/pdf/rca/v47n1/0045-6888-rca-47-01-0213.pdf >. Acesso em: Ago. 2021.

Negrelle, R. R. B. Composição e estrutura do componente arbóreo de mata com acuri no pantanal matogrossense, Brasil. Ciência Florestal, Santa Maria, v. 26, n. 2, p. 589-600, abr.jun., 2016.

Palazzolo, E. et al. Current and Potential Use of Citrus Essential Oils - Current Organic Chemistry. 2013, 17, 3042-3049. Disponível em: < https://core.ac.uk/download/pdf/53292733.pdf >. Acesso em: Ago. 2021.

Raij, B. van; Andrade, J. C. de; Cantarella, H.; Quaggio, J.A. Análise Química para Avaliação da Fertilidade de Solos Tropicais. Campinas, Instituto Agronômico, 285p. 2001

Raimundo, K. F. et al. Chemical composition of garlic wood (Gallesia integrifolia) (Phytolaccaceae) volatile compounds and their activity on cattle tick. AJCS 11(08):10581067 (2017). http://doi: 10.21475/ajcs.17.11.08.pne632

Raimundo, K. F. et al. Antifungal activity of Gallesia integrifolia fruit essential oil. Braz. J. Microbiol. 49: 229-235. 2018. https://doi.org/10.1016/j.bjm.2018.03.006 
Raimundo, K. F. et al. Insecticidal activity of Gallesia integrifolia (Phytolaccaceae) essential oil. Bol Latinoam Caribe Plant Med Aromat 20 (1): 38 - 50 (2021). https://doi.org/10.37360/blacpma.21.20.1.3

Rebey, I. B.; Jabri-Karoui, I.; Hamrouni-Sellami, I. et al. (2012). Effect of drought on the biochemical composition and antioxidant activities of cumin (Cuminum cyminum L.) seeds. Ind Crops Prod 36:238-245. doi:10.1016/j.indcrop.2011.09.013

Rodrigues, V. E. G.; Carvalho, D. A. Plantas medicinais no domínio dos cerrados. Lavras: UFLA/FAEPE, 2001. 180

p.

Simões, C. M. O. et al. Farmacognosia: da planta ao medicamento. 5 ed. Porto Alegre/Florianópolis: Editora da UFRGS / Editora UFSC, 2003

Sfredo, G. J.; Borkert, C. M. Deficiências e toxidades de nutrientes em plantas de soja. Londrina: Embrapa Soja, 2004

Sobral, L. S. et al. Guia prático para interpretação de resultados de análises de solos. Aracaju: Embrapa Tabuleiros Costeiros, 2015. 13 p. Disponível em: <https://ainfo.cnptia.embrapa.br/digital/bitstream/item/142260/1/Doc-206.pdf >. Acesso em: Ago. 2021.

Teixeira, I. R. et al. Teores de nutrientes e qualidade fisiológica de sementes de feijão em resposta à adubação foliar com manganês e zinco. Campinas, Bragantia, v.64, n.1, p.8388, 2005. Disponível em: < http://www.scielo.br/pdf/\%0D/brag/v64n1/23855.pdf >. Acesso em: Ago. 2021.

Viégas, I. D. J. M. et al. Efeito da omissão de macronutrientes e boro no crescimento, nos sintomas de deficiências nutricionais e na composição mineral de plantas de camucamuzeiro. Jaboticabal - SP, Rev. Bras. Frutic., v. 26, n. 2, p. 315-319, Ago. 2004. Disponível em: < http://www.scielo.br/pdf/\%0D/rbf/v26n2/21833.pdf >. Acesso em: Ago. 2021.

Villar, M. L. P. Manual de interpretação de análise de plantas e solos e recomendação de adubação. Cuiabá: EMPAER-MT, 2007. 182 p. (EMPAER-MT, Série Documentos, 35). Disponívelem:<http://www.empaer.mt.gov.br/documents/8024815/9382198/Manual+de+ Interpreta\%C3\% A7\% C3\%A3o+de+An\%C3\%A1lise+de+Plantas+e+Solos+e+Recomend a\%C3\%A7\%C3\%A3o+de+Aduba\%C3\%A7\%C3\%A3o/09cae279-bdf6-5b4a-4b0567fe9b4522f1>. Acesso em: Ago. 2021.

Zambolim, L.; Ventura, J. A. resistência a doenças induzida pela nutrição mineral das plantas. Revisão Anual de Patologia de Plantas. Passo Fundo: RAPP, v.1, p. 275-319,1993. Disponível em: <https://biblioteca.incaper.es.gov.br/digital/bitstream/item/2360/1/BRTresistenciaado encasinduzidapelanutricaomineraldasplantas-Emcapa.pdf >. Acesso em: Ago. 2021. 